\title{
BMJ Open Prognostic value of reduction in left atrial size during a follow-up of heart failure: an observational study
}

Masayuki Shiba, ${ }^{1}$ Takao Kato (D) , , Takeshi Morimoto (1) , ${ }^{2}$ Hidenori Yaku, ${ }^{3}$ Yasutaka Inuzuka, ${ }^{4}$ Yodo Tamaki, ${ }^{5}$ Neiko Ozasa, ${ }^{1}$ Yuta Seko, ${ }^{1}$ Erika Yamamoto, ${ }^{1}$ Yusuke Yoshikawa, ${ }^{1}$ Takeshi Kitai, ${ }^{6}$ Yugo Yamashita, ${ }^{1}$ Moritake Iguchi (D) ,7 Kazuya Nagao (D) , ${ }^{8}$ Yuichi Kawase, ${ }^{9}$ Takashi Morinaga, ${ }^{10}$ Mamoru Toyofuku, ${ }^{11}$ Yutaka Furukawa, ${ }^{6}$ Kenji Ando, ${ }^{10}$ Kazushige Kadota, ${ }^{9}$ Yukihito Sato, ${ }^{12}$ Koichiro Kuwahara, ${ }^{13}$ Takeshi Kimura (D) , ${ }^{1}$ On behalf of KCHF Study Investigators

To cite: Shiba M, Kato T, Morimoto T, et al. Prognostic value of reduction in left atrial size during a followup of heart failure: an observational study. BMJ Open 2021;11:e044409. doi:10.1136/ bmjopen-2020-044409

- Prepublication history and additional material for this paper is available online. To view these files, please visit the journal online (http://dx.doi.org/10. 1136/bmjopen-2020-044409).

Received 02 September 2020 Revised 26 January 2021 Accepted 04 February 2021
Check for updates

(C) Author(s) (or their employer(s)) 2021. Re-use permitted under CC BY-NC. No commercial re-use. See rights and permissions. Published by BMJ.

For numbered affiliations see end of article.

Correspondence to

Dr Takao Kato;

tkato75@kuhp.kyoto-u.ac.jp

\section{ABSTRACT}

Objective The association between sequential changes in left atrial diameter (LAD) and prognosis in heart failure (HF) remains to be elucidated. The present study aimed to investigate the link between reduction in $L A D$ and clinical outcomes in patients with HF.

Design A multicentre prospective cohort study.

Setting This study was nested from the Kyoto Congestive Heart Failure registry including consecutive patients admitted for acute decompensated heart failure (ADHF) in 19 hospitals throughout Japan.

Participants The current study population included 673 patients with HF who underwent both baseline and 6-month follow-up echocardiography with available paired $L A D$ data. We divided them into two groups: the reduction in the LAD group (change $<0 \mathrm{~mm})(\mathrm{n}=398)$ and the noreduction in the LAD group (change $\geq 0 \mathrm{~mm})(\mathrm{n}=275)$.

Primary and secondary outcomes The primary outcome measure was a composite of all-cause death or hospitalisation for HF during 180 days after 6-month follow-up echocardiography. The secondary outcome measures were defined as the individual components of the primary composite outcome measure and a composite of cardiovascular death or hospitalisation for HF.

Results The cumulative 180-day incidence of the primary outcome measure was significantly lower in the reduction in the LAD group than in the no-reduction in the LAD group $(13.3 \%$ vs $22.2 \%, p=0.002)$. Even after adjusting 15 confounders, the lower risk of reduction in LAD relative to no-reduction in LAD for the primary outcome measure remained significant (HR $0.59,95 \% \mathrm{Cl} 0.36$ to 0.97 $\mathrm{p}=0.04$ ).

Conclusion Patients with reduction in $L A D$ during follow-up after ADHF hospitalisation had a lower risk for a composite endpoint of all-cause death or HF hospitalisation, suggesting that the change of LAD might be a simple and useful echocardiographic marker during follow-up.

\section{INTRODUCTION}

The prevalence of heart failure (HF) and the number of $\mathrm{HF}$ hospitalisations have been increasing worldwide with an ageing

\section{Strengths and limitations of this study}

- This study is a multicentre prospective observational study reporting the association between sequential changes in left atrial diameter (LAD) and prognosis in heart failure (HF).

- We calculated changes in LAD from the echocardiography during index hospitalisation to 6-month follow-up echocardiography.

- A link between reduction in LAD and favourable clinical outcomes was demonstrated even after adjusting for left atrial enlargement.

- The study results were derived from very selected patients from the Kyoto Congestive Heart Failure registry participants.

- Further studies are needed to generalise our study results.

population. ${ }^{1}$ Further, the health expenditure for $\mathrm{HF}$ is progressively increasing, and $\mathrm{HF}$ is a crucial global health problem. ${ }^{2} \mathrm{HF}$ hospitalisations are often repeated, ${ }^{3}$ and quality of life and outcomes in HF progressively deteriorate with HF finally leading to death. ${ }^{4}$ Thus, the outpatient management of $\mathrm{HF}$ is critically important. However, there are very limited data on the association between longitudinal echocardiographic changes over time and clinical outcomes in patients with HF. ${ }^{5}$ Left atrial enlargement is an established prognostic predictor of $\mathrm{HF}^{6}$ and is related to mortality in heart diseases. ${ }^{78}$ Besides, left atrial enlargement was independently associated with cardiovascular events and death in the general population. ${ }^{9}$ Despite the prognostic importance of left atrial size, the relation between sequential change in left atrial size and the prognosis in HF has not been evaluated. 
In this study, we evaluated left atrial diameter (LAD) during 6 months after hospitalisation for acute decompensated heart failure (ADHF) and investigated the effect of the reduction in LAD on clinical outcomes in patients who were discharged from ADHF hospitalisation.

\section{METHODS}

\section{Patient population}

In the Kyoto Congestive Heart Failure (KCHF) registry, we enrolled consecutive 4056 hospitalised patients who were diagnosed as ADHF by the modified Framingham criteria and were treated with HF-specific management involving intravenous drugs within 24 hours after hospital admittance between 1 October 2014 and 31 March 2016. The rationale, design and enrolment of the KCHF registry have been previously published in detail. ${ }^{10-12}$ In parallel with the main KCHF study, we designed a prospective longitudinal follow-up study enrolling patients who were to have a visit and echocardiography at $6 \pm 1$ months. ${ }^{10}$ At the 6 -month visit, we collected data on physical findings, echocardiography, laboratory tests and medications. Exclusion criteria for the prospective longitudinal follow-up study included the following: no consent given for the longitudinal study $(n=238)$, age $<20$ years $(n=1)$, fever or infectious diseases at admission $(n=297)$, acute coronary syndrome at admission $(n=157)$, end-stage renal failure $(n=218)$, severe comorbidity which limits the life expectancy within 1 year assessed by attending physicians at each participating centre, such as end-stage cancer, severe cognitive dysfunction, and end-stage liver dysfunction $(n=112)$, or ineligible for the protocol mandated follow-up by attending physicians' judgement $(n=1516)$. The prospective longitudinal follow-up cohort included 1246 patients after excluding 2539 patients who had exclusion criteria, and 271 patients who died during index hospitalisation. Follow-up echocardiography at the 6-month visit was performed in 748 patients, and after excluding those patients with missing LAD data, the current study population consisted of 673 patients who underwent both baseline and follow-up echocardiography with available paired LAD data (figure 1A,B). We calculated changes in LAD from the echocardiography during index hospitalisation to 6-month follow-up echocardiography and divided the study population into two groups: the reduction in the LAD group ( $n=398)$ and the no-reduction in the LAD group $(n=275)$.

Data on clinical characteristics and echocardiography were collected from hospital medical records. Clinical follow-up information was obtained from hospital medical records and/or from letters or telephone call to patients, their relatives or their referring physicians by the attending physicians or research assistants at each participating institution. Identifiable patient data were anonymised before analysis.

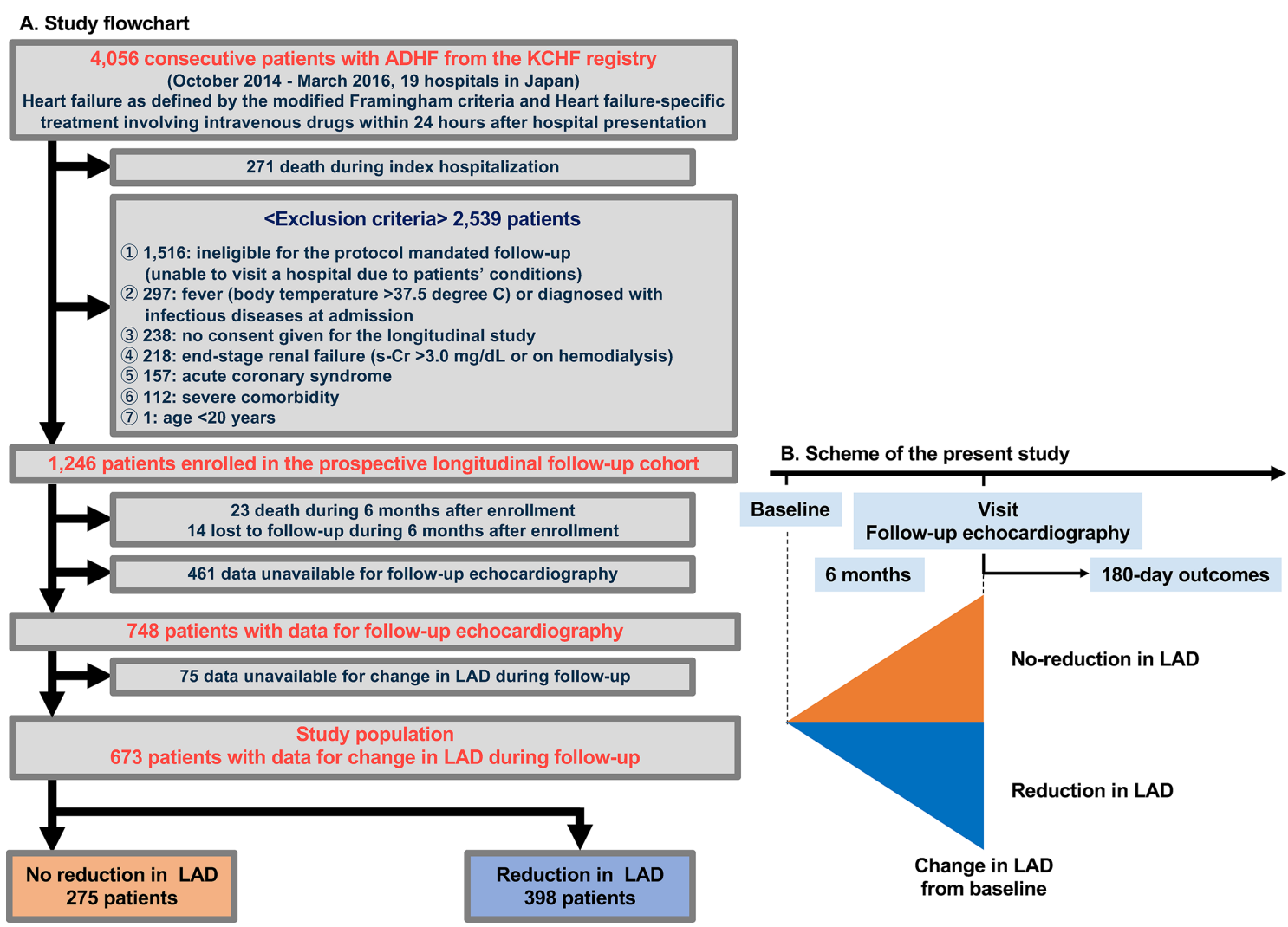

Figure 1 (A) Study flowchart. (B) A scheme of the present analysis. ADHF, acute decompensated heart failure; KCHF, Kyoto Congestive Heart Failure; LAD, left atrial diameter; s-Cr, serum creatinine. 


\section{Ethics}

The investigation conforms with the principles outlined in the Declaration of Helsinki Written informed consent was obtained from patients enrolled in the longitudinal prospective cohort study.

\section{Outcomes}

Clinical follow-up was performed at 1 year \pm 1 month after enrolment; thus, the data were censored at 210 days after the follow-up echocardiography at 6 months after index hospitalisation and we performed time-to-event analyses. The date of follow-up echocardiography was regarded as time zero (figure 1B). The primary outcome measure in the present study was defined as a composite of allcause death or hospitalisation for HF. The secondary outcome measures were defined as the individual components of the primary composite outcome measure and a composite of cardiovascular death or hospitalisation for HF. Death was regarded as cardiovascular in origin unless obvious non-cardiovascular causes could be identified. ${ }^{1011}$ Cardiovascular death included death related to HF, sudden death, death related to stroke and death from other cardiovascular causes. Sudden death was an unexplained death in a previously stable patient. ${ }^{10} 11$

\section{Definitions}

LAD was measured as anteroposterior diameter of left atrium during systolic phase in parasternal long-axis view by echocardiography. We did not adopt the data for LA measurement in other views. We defined a reduction in LAD from baseline to 6-month follow-up echocardiography as $\mathrm{LAD}$ change $<0 \mathrm{~mm}$ and a no-reduction in LAD as LAD change $\geq 0 \mathrm{~mm}$.

The detailed definitions of baseline patient characteristics were previously described. ${ }^{10-15}$ Anaemia was defined using the WHO criteria (haemoglobin $<120 \mathrm{~g} / \mathrm{L}$ in women and $<130 \mathrm{~g} / \mathrm{L}$ in men). End-stage renal disease was defined as estimated glomerular filtration rate $<30 \mathrm{~mL} /$ $\min / 1.73 \mathrm{~m}^{2}$ based on the chronic kidney disease grades. $\mathrm{HF}$ was classified according to left ventricular ejection fraction (LVEF) at 6-month follow-up as $\mathrm{HF}$ with reduced LVEF $(<40 \%)$ (HFrEF) and non-HF with reduced LVEF $(\geq 40 \%)$ (non-HFrEF). In addition, we classified $\mathrm{HF}$ according to $\mathrm{LVEF} \geq 50 \%$ (HF with preserved $\mathrm{EF}$ ), $40 \%-49 \%$ (HF with mid-range EF) and $<40 \%$ (HFrEF). Left atrial enlargement was defined as LAD $\geq 40 \mathrm{~mm}^{7}$ $\mathrm{LV}$ dilation was defined as left ventricular end-diastolic dimension (LVDd) $>55 \mathrm{~mm} .{ }^{16}$ High tricuspid regurgitation pressure gradient (TRPG) was defined as TRPG $>31.4 \mathrm{~mm} \mathrm{Hg}^{17}$

\section{Statistical analysis}

Categorical variables were expressed as counts and percentages, and continuous variables were expressed as means with SD, or medians with IQR. A $\chi^{2}$ test was used for categorical variables. An unpaired, two-tailed t-test was used for normally distributed continuous variables or the Wilcoxon rank-sum test was used for non-normally distributed continuous variables. Baseline patient characteristics and clinical outcomes were compared between the two groups based on LAD change patterns. A paired t-test was used for continuous variables and sign test was used for binary variables to compare them between baseline and follow-up. Cumulative incidences were estimated by Kaplan-Meier analysis and the between-groups differences were assessed by log-rank test. We used the Cox proportional hazards regression model to estimate the effect of reduction in $\mathrm{LAD}$ relative to no-reduction in $\mathrm{LAD}$ for the primary and secondary outcome measures incorporating 15 clinically relevant risk-adjusting variables including age $\geq 80$ years, sex, atrial fibrillation/flutter, renal failure, anaemia, $\mathrm{LVEF}<40 \%$, moderate/severe mitral regurgitation (MR), LAD $\geq 40 \mathrm{~mm}$, TRPG $>31.4 \mathrm{~mm} \mathrm{Hg}$, change in TRPG $>0 \mathrm{~mm} \mathrm{Hg}$ LVDd $>55 \mathrm{~mm}$, change in LVDd $>0 \mathrm{~mm}$, angiotensin-converting enzyme inhibitor (ACE-I) or angiotensin-receptor blocker, $\beta$-blockers and mineralocorticoid receptor antagonist. The results were presented as the HRs and their $95 \%$ CIs. The subgroup analyses were performed in the 10 clinically relevant subgroups such as age equal to or greater than 80 years, sex, atrial fibrillation/flutter, moderate/severe MR, LVEF less than $40 \%$, and LAD equal to or greater than $40 \mathrm{~mm}$, TRPG higher than $31.4 \mathrm{~mm} \mathrm{Hg}$, change in TRPG higher than $0 \mathrm{~mm} \mathrm{Hg}$, LVDd $>55 \mathrm{~mm}$ and change in LVDd $>0 \mathrm{~mm}$. We also used the Cox proportional hazards regression model to estimate the interactions between the subgroup factors and the effect of LAD change patterns on clinical outcomes. Additionally, factors associated with reduction in LAD were analysed using univariate and multivariate logistic regression models, adjusting for 17 clinically relevant variables. The 17 adjusting variables consisted of change in LVDd $>0 \mathrm{~mm}$, change in LVEF $>0 \%$, change in TRPG $>0 \mathrm{~mm} \mathrm{Hg}$, data at discharge from index hospitalisation which correspond to the risk-adjusting variables of the main analysis and diuretics at discharge. Statistical analyses were performed using JMP pro software, V.14 (SAS). A two-tailed $\mathrm{p}$ value $<0.05$ was considered as statistically significant in all analyses.

\section{Patient and public involvement}

Patients and/or the public were not involved in the design, or conduct, or reporting or dissemination plans of this research.

\section{RESULTS \\ Clinical characteristics, laboratory test results and medications at the 6-month visit}

The clinical characteristics at the 6-month visit were well balanced between the two groups, except for the lower prevalence of patients with age $\geq 80$ years in the reduction in the LAD group than in the no-reduction in the LAD group (table 1). The laboratory test results and medications were also well balanced between the two groups, except for the higher haemoglobin level and lower B-type natriuretic polypeptide level in the reduction in the 
Table 1 Patient characteristics at 6-month echocardiographic follow-up

\begin{tabular}{|c|c|c|c|c|}
\hline Variable & No reduction in LAD $(n=275)$ & Reduction in LAD $(n=398)$ & P value & $\begin{array}{l}\text { Number of patients } \\
\text { analysed }\end{array}$ \\
\hline \multicolumn{5}{|l|}{ Clinical characteristics } \\
\hline Age (years) & $76.1 \pm 11.6$ & $74.2 \pm 12.6$ & 0.07 & 673 \\
\hline Age $\geq 80$ years* & $130(47 \%)$ & $154(39 \%)$ & 0.03 & 673 \\
\hline Women* & $130(47 \%)$ & $165(41 \%)$ & 0.14 & 673 \\
\hline \multicolumn{5}{|l|}{ Medical history } \\
\hline Atrial fibrillation or flutter & $156(57 \%)$ & $222(56 \%)$ & 0.81 & 673 \\
\hline Hypertension & $212(77 \%)$ & $287(72 \%)$ & 0.15 & 673 \\
\hline Diabetes & $115(42 \%)$ & $141(35 \%)$ & 0.09 & 673 \\
\hline eGFR (mL/min/1.73 m²) & $43.4 \pm 19.4$ & $46.5 \pm 20.8$ & 0.06 & 642 \\
\hline eGFR $<30 \mathrm{~mL} / \mathrm{min} / 1.73 \mathrm{~m}^{2 \star}$ & $71(27 \%)$ & $86(23 \%)$ & 0.23 & 642 \\
\hline Albumin (g/dL) & $3.9 \pm 0.46$ & $3.9 \pm 0.57$ & 0.24 & 608 \\
\hline Albumin $<3 \mathrm{~g} / \mathrm{dL}$ & $7(2.8 \%)$ & $16(4.5 \%)$ & 0.26 & 608 \\
\hline Haemoglobin (g/L) & $118 \pm 22$ & $121 \pm 23$ & 0.03 & 638 \\
\hline Anaemia * & $169(64 \%)$ & $208(56 \%)$ & 0.03 & 638 \\
\hline \multicolumn{5}{|l|}{ Medications at 6-month follow-up } \\
\hline ACE-I or $\mathrm{ARB}^{*}$ & $139(60 \%)$ & $194(62 \%)$ & 0.70 & 545 \\
\hline
\end{tabular}

Diuretics included loop diuretic, thiazide and tolvaptan.

${ }^{*}$ Risk-adjusting variables selected for the Cox proportional hazards regression model.

ACE-I, angiotensin-converting enzyme inhibitor; ARB, angiotensin-receptor blocker; BMI, body mass index; BNP, brain natriuretic peptide; eGFR, estimated glomerular filtration rate; $L A D$, left atrial diameter; MRA, mineralocorticoid receptor antagonist.

LAD group than in the no-reduction in the LAD group (table 1).

\section{Echocardiographic findings}

The reduction in the LAD group had a larger LAD, larger LVDd and higher TRPG at baseline echocardiography than the no-reduction in the LAD group. At 6-month follow-up echocardiography, TRPG was smaller in the reduction in the LAD group than in the no-reduction in the LAD group (table 2). Between baseline and 6-month follow-up echocardiography, decrease in TRPG and decrease in the prevalence of moderate/severe MR and tricuspid regurgitation were observed only in the reduction in the LAD group. The magnitudes of the decrease in LVDd, the decrease in TRPG and the increase of LVEF were greater in the reduction in the $\mathrm{LAD}$ group than in the no-reduction in the LAD group (table 2).
Clinical outcomes: the reduction in the LAD group versus the no-reduction in the LAD group

The follow-up rate at 180 days after 6-month follow-up echocardiography was $96.3 \%$. The cumulative 180-day incidence of the primary outcome measure (a composite endpoint of all-cause death or hospitalisation for HF) was significantly lower in the reduction in the LAD group than in the no-reduction in the LAD group ( $13.3 \%$ vs $22.2 \%$, $\mathrm{p}=0.002$ ) (figure 2A). After adjusting for confounders, the lower risk of the reduction in the LAD group relative to the no-reduction in the LAD group for the primary outcome measure remained significant (HR 0.59, $95 \%$ CI 0.36 to $0.97 \mathrm{p}=0.04$ ) (table 3 ). Regarding the secondary outcome measures, the cumulative 180-day incidence of all-cause death was also significantly lower in the reduction in the $\mathrm{LAD}$ group than in the no-reduction in the $\mathrm{LAD}$ group $(4.6 \%$ vs $8.6 \%, \mathrm{p}=0.01$ ) (figure $2 \mathrm{~B}$ ). After adjusting for confounders, the lower risk of the reduction in the $\mathrm{LAD}$ group relative to the no-reduction in the 


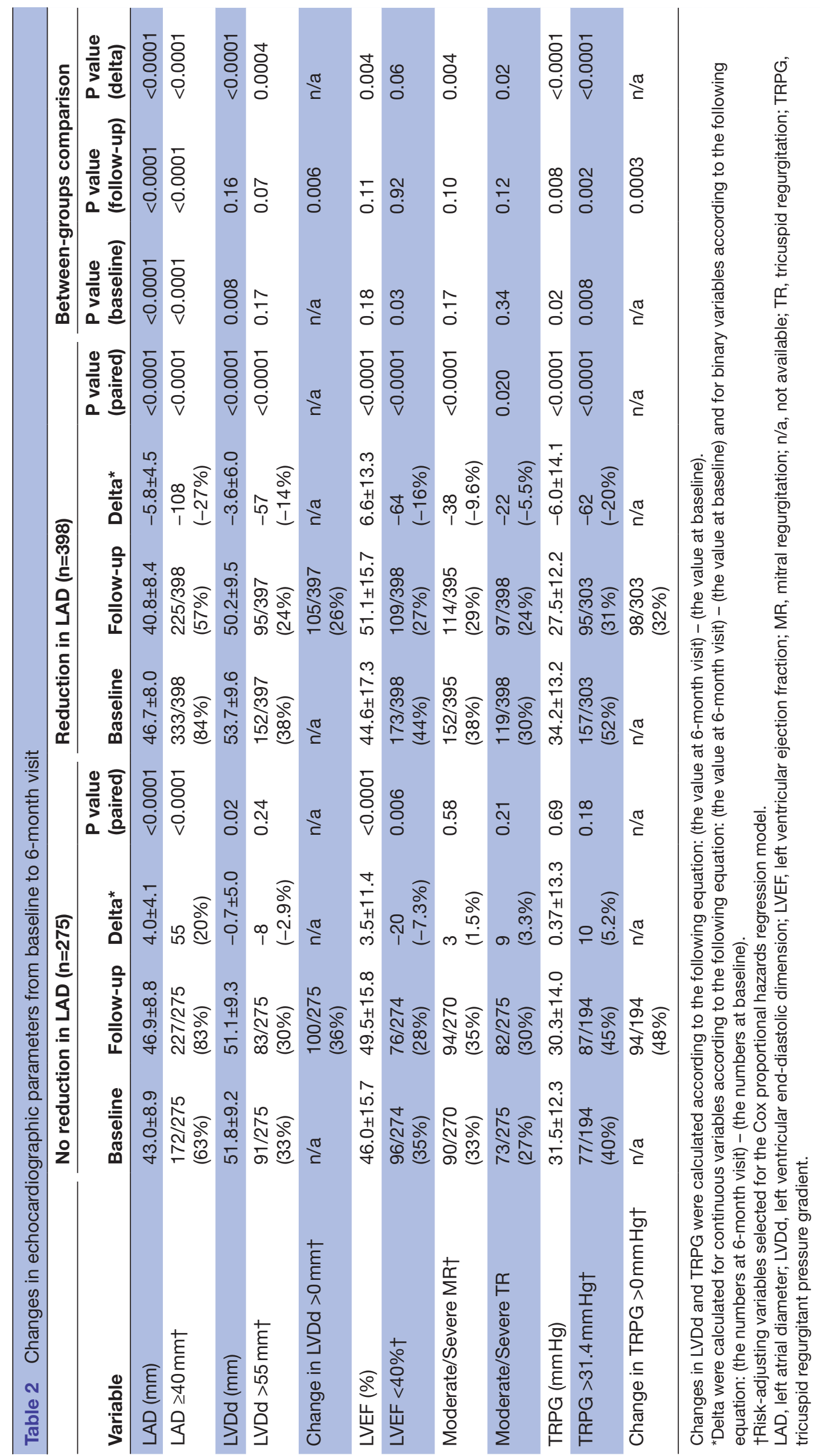



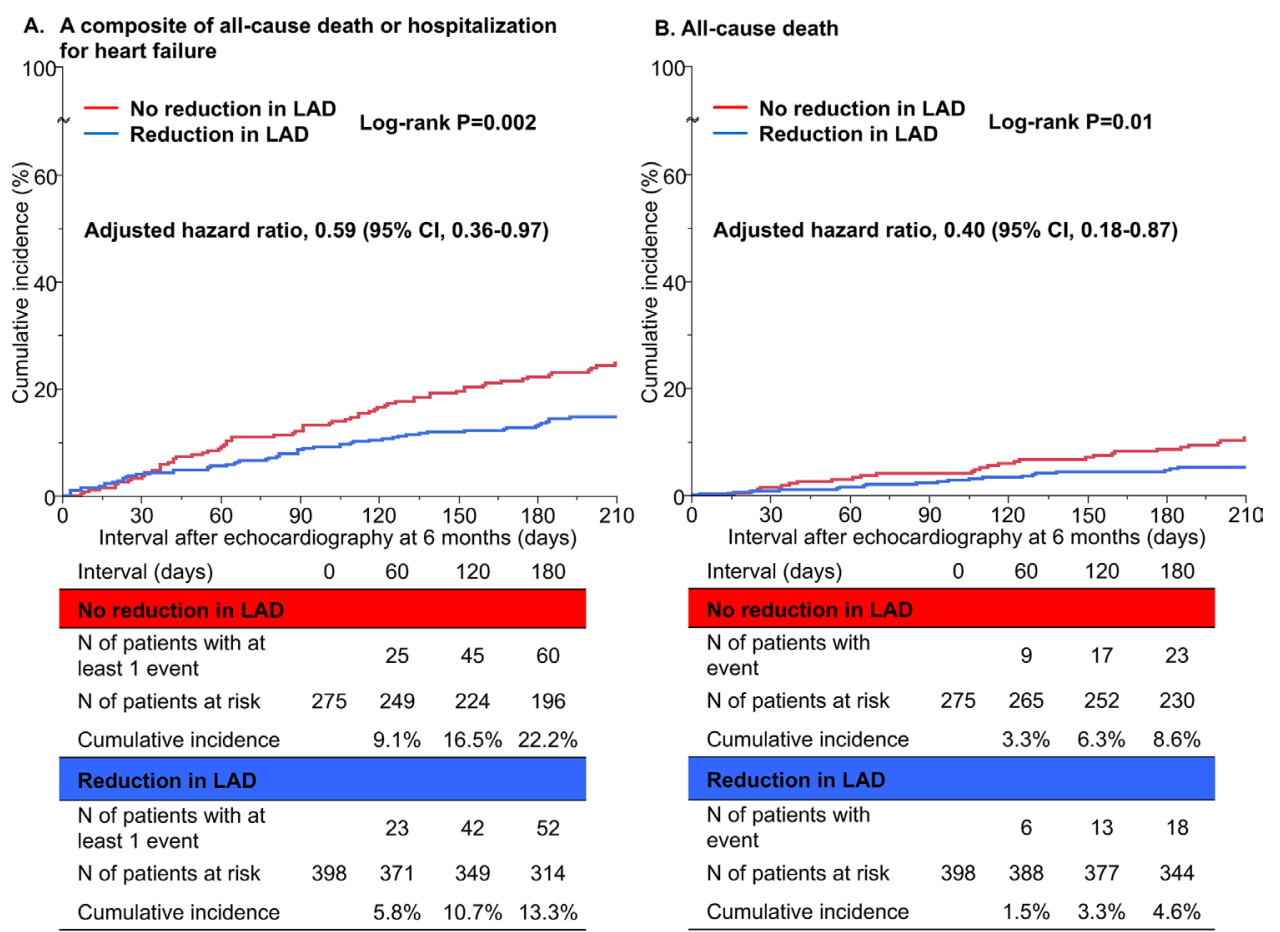

C. Hospitalization for heart failure
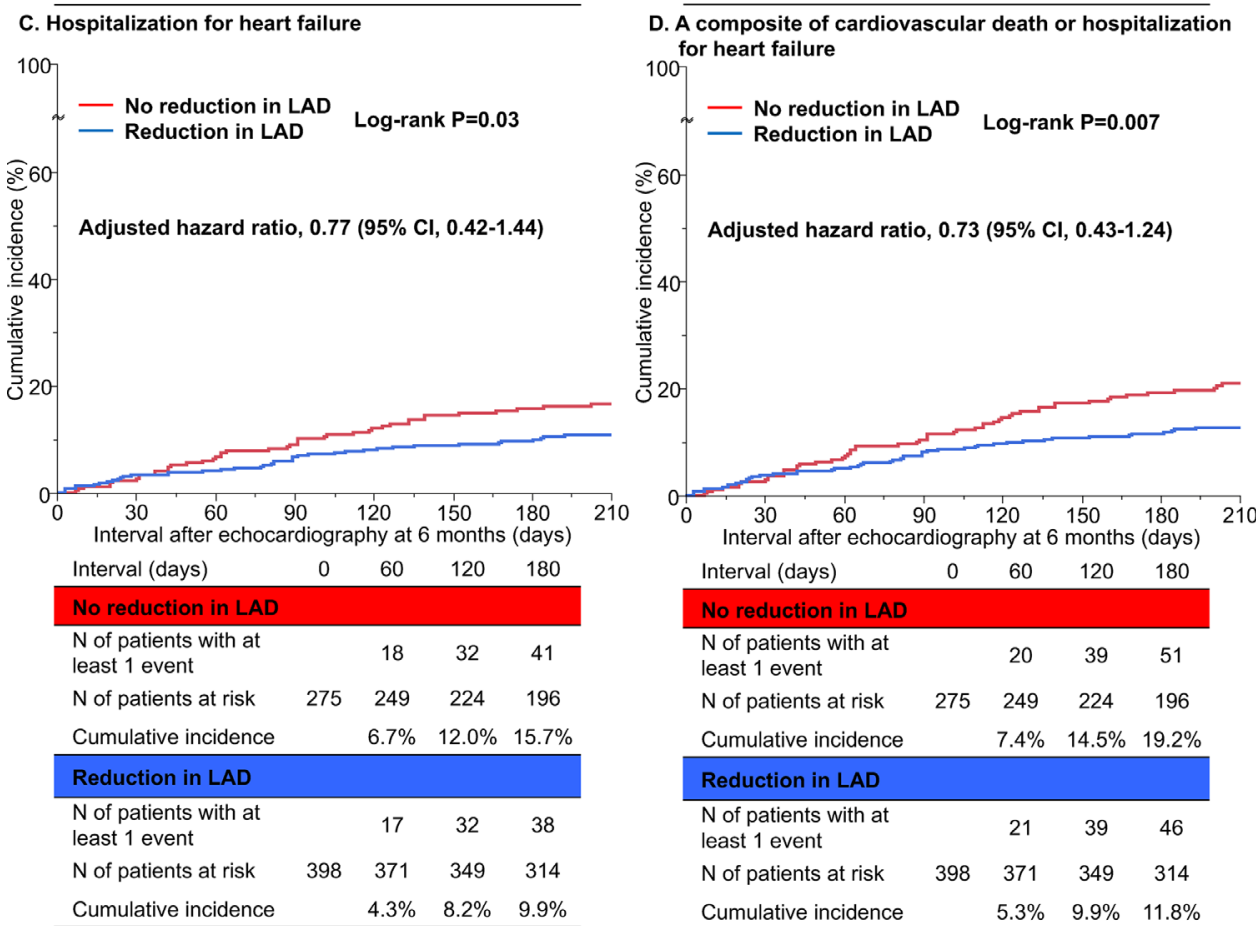

Figure 2 Kaplan-Meier curves for (A) the primary outcome measure, (B) all-cause death, (C) hospitalisation for heart failure and (D) a composite of cardiovascular death or hospitalisation for heart failure. LAD, left atrial diameter.

LAD group for all-cause death remained significant (HR $0.40,95 \%$ CI 0.18 to $0.87, \mathrm{p}=0.02$ ) (table 3 ). The cumulative 180-day incidences of hospitalisation for $\mathrm{HF}$ and a composite of cardiovascular death or hospitalisation for HF were also significantly lower in the reduction in the $\mathrm{LAD}$ group than in the no-reduction in the LAD group (9.9\% vs $15.7 \%, \mathrm{p}=0.03$, figure $2 \mathrm{C}$ and $11.8 \%$ vs $19.2 \%$, $\mathrm{p}=0.007$, figure $2 \mathrm{D}$, respectively). However, after adjusting for confounders, the lower risk of the reduction in the $\mathrm{LAD}$ group relative to the no-reduction in the LAD group for hospitalisation for $\mathrm{HF}$ and a composite of cardiovascular death or hospitalisation for $\mathrm{HF}$ were no longer significant (HR $0.77,95 \%$ CI 0.42 to $1.44, p=0.42$ and HR $0.73,95 \%$ CI 0.43 to $1.24, \mathrm{p}=0.24$, respectively) (table 3 ).

\section{Subgroup analyses}

When we stratified patients according to the subgrouping factors, the reduction in LAD was greater in patients aged below 80 years, men, and patients without MR, $\mathrm{LAD}>40 \mathrm{~mm}$, or an increase in TRPG and LVDd (online 
Table 3 Clinical outcomes at 180 days after follow-up echocardiography

\section{Number of patients with event/Number \\ of patients at risk \\ (cumulative 180-day incidence)}

HR $(95 \% \mathrm{Cl})$

\section{Reduction in LAD No reduction in LAD}

\begin{tabular}{|c|c|c|c|c|c|c|}
\hline Clinical outcome measures & $(n=398)$ & $(n=275)$ & Crude & $P$ value & Adjusted & $P$ value \\
\hline $\begin{array}{l}\text { All-cause death or } \\
\text { hospitalisation for heart failure }\end{array}$ & $52 / 314(13.3 \%)$ & $60 / 196(22.2 \%)$ & $\begin{array}{l}0.57 \\
(0.40 \text { to } 0.81)\end{array}$ & 0.002 & $\begin{array}{l}0.59 \\
(0.36 \text { to } 0.97)\end{array}$ & 0.04 \\
\hline All-cause death & $18 / 344(4.6 \%)$ & $23 / 230(8.6 \%)$ & $\begin{array}{l}0.48 \\
(0.27 \text { to } 0.85)\end{array}$ & 0.01 & $\begin{array}{l}0.40 \\
(0.18 \text { to } 0.87)\end{array}$ & 0.02 \\
\hline $\begin{array}{l}\text { Hospitalisation for heart } \\
\text { failure }\end{array}$ & $38 / 314$ (9.9\%) & $41 / 196(15.7 \%)$ & $\begin{array}{l}0.63 \\
(0.41 \text { to } 0.97)\end{array}$ & 0.04 & $\begin{array}{l}0.77 \\
(0.42 \text { to } 1.44)\end{array}$ & 0.42 \\
\hline $\begin{array}{l}\text { Cardiovascular death or } \\
\text { hospitalisation for heart failure }\end{array}$ & 46/314 (11.8\%) & $51 / 196(19.2 \%)$ & $\begin{array}{l}0.59 \\
(0.40 \text { to } 0.87)\end{array}$ & 0.007 & $\begin{array}{l}0.73 \\
(0.43 \text { to } 1.24)\end{array}$ & 0.24 \\
\hline
\end{tabular}

The Cox proportional hazards regression model was constructed adjusting for 15 clinically relevant risk-adjusting variables: age $\geq 80$ years, sex, atrial fibrillation/flutter, renal failure, anaemia, LVEF $<40 \%$, moderate/severe MR, LAD $\geq 40 \mathrm{~mm}$, TRPG $>31.4 \mathrm{~mm} \mathrm{Hg}$, change in TRPG $>0 \mathrm{~mm} \mathrm{Hg}$, LVDd $>55 \mathrm{~mm}$, change in LVDd $>0 \mathrm{~mm}$, ACE-I or ARB, $\beta$-blockers and MRA.

ACE-I, angiotensin-converting enzyme inhibitor; ARB, angiotensin-receptor blocker; LAD, left atrial diameter; LVDd, left ventricular enddiastolic dimension; LVEF, left ventricular ejection fraction; MR, mitral regurgitation; MRA, mineralocorticoid receptor antagonist; TRPG, tricuspid regurgitation pressure gradient.

supplemental table 1). In the subgroup analyses, there were no significant interactions between all the subgroup factors (age, sex, atrial arrythmias, moderate/severe MR, reduced LVEF, LAD enlargement, high TRPG and its change, LV dilation and its change) and the effects of reduction in $\mathrm{LAD}$ relative to no-reduction in $\mathrm{LAD}$ on the primary outcome measure (online supplemental figure 1 ). When stratified by LVEF $\geq 50 \%, 40 \%-49 \%$ and $<40 \%$, there was no interaction between the LVEF status and the effect in reduction in LAD (online supplemental table 2).

\section{Factors associated with the reduction in LAD}

Among the variables listed in online supplemental table 3 , the following variables were significantly associated with reduction in $\mathrm{LAD}$ in univariate logistic regression analysis: age $\geq 80$ years, $\mathrm{LAD} \geq 40 \mathrm{~mm}$, change in $\mathrm{LVDd}>0 \mathrm{~mm}$, LVEF $<40 \%$, change in LVEF $>0 \%$, TRPG $>31.4 \mathrm{~mm} \mathrm{Hg}$ and change in TRPG $>0 \mathrm{~mm} \mathrm{Hg}$ (online supplemental table 4). In multivariate logistic regression analysis, $\mathrm{LAD} \geq 40 \mathrm{~mm}$, TRPG $>31.4 \mathrm{~mm} \mathrm{Hg}$ and change in TRPG $>0 \mathrm{~mm} \mathrm{Hg}$ were significantly associated with the reduction in LAD (online supplemental table 4).

\section{DISCUSSION}

The main findings of the present study are as follows: (1) a reduction in $\mathrm{LAD}$ during 6 -month follow-up after $\mathrm{ADHF}$ hospitalisation was associated with echocardiographic parameters such as large LA at baseline, an increase in LVEF and a decrease in both LVDd and TRPG; and (2) a reduction in $\mathrm{LAD}$ during 6-month follow-up was associated with a lower risk for a composite of all-cause death or hospitalisation for HF.

Many studies reported that left atrial enlargement was a very useful prognostic marker in patients with heart disease including HF. ${ }^{8}{ }^{18-20}$ However, they assessed left atrial size at a single time point, not serially. Meris $e t a l^{21}$ investigated the association of the change of left atrial volume indexed to body surface area with clinical outcomes in patients with acute myocardial infarction. However, no previous study researched the association between sequential change in left atrial size and clinical outcomes in patients with HF. In clinical settings, we serially assess the cardiac structure and function by echocardiography during follow-up. In addition, $\mathrm{LAD}$ is a fundamental measurement of echocardiogram ${ }^{22}{ }^{23}$; thus, changes of LAD can be easily evaluated. In the present study, we showed that the change of left atrial size was associated with the prognosis in patients with HF regardless of the presence of left atrial enlargement.

The differences between the reduction and no-reduction in the LAD groups in echocardiographic findings might indicate that more LV reverse remodelling occurred in the reduction in the LAD group. Functionally, LA modulates LV filling and cardiovascular performance as a reservoir for pulmonary venous inflow during LV systole, a conduit for pulmonary venous flow during early ventricular diastole and an active pump to aid LV filling during late ventricular diastole. ${ }^{18}$ LA is structurally and functionally correlated with LV function. ${ }^{24}{ }^{25}$ In particular, LA size is closely linked to LV filling pressure, indicating the change in LA size would be correlated to the change in LV filling pressure. ${ }^{24-26}$ In our study, the increase of LVEF was greater in the reduction in the LAD group than in the no-reduction in the LAD group. Thus, the improved LV systolic function in the reduction in the LAD group might be attributed to decrease in LV volume and LV filling pressure. In addition, a reduction in LA size and a decrease in the prevalence of moderate/severe MR and TRPG in the reduction in the LAD group might be related to 
improvement of LV diastolic dysfunction. Altogether, a reduction in LAD suggestive of LA reverse remodelling might be an indicator of the recovery from diastolic and systolic LV dysfunction.

In our study, patients with a reduction in LAD during 6-month follow-up after ADHF hospitalisation showed a better outcome than those with no-reduction in LAD. After adjusting for confounders including medications for HF, LA reverse remodelling was independently associated with a lower risk for a composite of all-cause death or hospitalisation for HF. LV dilation/remodelling is the conventional risk factor for $\mathrm{HF}$ and mortality. ${ }^{27}{ }^{28} \mathrm{LVEF}$ was correlated to mortality in patients with chronic HF. ${ }^{29}$ Echocardiographic findings in index hospitalisation suggested a worse condition in the reduction in the LAD group with larger LA, larger LV, higher TRPG and higher prevalence of reduced LVEF. Although remodelling may not be similar in different types of HF aetiologies, improved outcome associated with reduction in LAD was consistently observed regardless of the presence of LV systolic dysfunction. LV reverse remodelling with increase of LVEF and decrease of LVDd was more frequently observed in the reduction in the LAD group than in the no-reduction in the LAD group. A definitive conclusion could not be drawn because of the limited number of patients analysed in subgroup; however, LV reverse remodelling might have contributed to improved outcome in the reduction in the LAD group.

Several studies on the change in LA volume index were derived from the data of patients with myocardial infarction or those with a risk factor of HF. ${ }^{21} 30$ The present study population consisted of patients who experienced hospitalisation(s) for ADHF. Thus, more elderly patients with more chronic comorbidities such as chronic kidney disease, chronic lung disease and atrial fibrillation were included..$^{21}{ }^{30}$ Despite the presence of many poor prognostic factors for HF, our study showed that reduction in LAD was independently associated with a significant reduction in the primary composite outcome measure, and all-cause death, as well as a trend for reduction in HF hospitalisation and a composite of cardiovascular death or hospitalisation for HF. Further studies are needed to combine the changes in various chamber sizes and other echocardiographic parameters together in one model and see which is the most important predictor of better clinical outcomes in these patients.

\section{Limitations}

Several limitations of the present study should be noted. First, two most recent chamber quantification guidelines recommend the use of left atrial volume assessment to quantify left atrial size instead of anteroposterior diameter, although we did not collect the left atrial volume. ${ }^{22}{ }^{23}$ LAD is not enough to measure left atrial size in patients with HF. This is a strong limitation of the present study. In addition, the echocardiographic data were not analysed by an echocardiography core laboratory, but by each participating institution. There might be interobserver and intraobserver differences in the measurement of LAD, although it is easy and fundamental in echocardiography. Diagnostic accuracy of LA measurement by echocardiography was validated when compared with that of CT or cardiac MRI. ${ }^{22} 23$ Second, we did not collect the echocardiographic data on cardiac output and diastolic function. We collected the peak early (E) and late (A) diastolic transmitral flow velocity and E/A ratio in patients with sinus rhythm, while Doppler early diastolic mitral annular velocity (e') and other surrogate markers of diastolic dysfunction were not collected in the present study. ${ }^{17}$ Furthermore, although LA and LV global longitudinal strain are useful for evaluating patients with $\mathrm{HF}^{31}{ }^{32}$ speckled tracking was not performed in this study. Third, it is possible that absent data can alter the study results (ie, selection bias); many patients ineligible for the protocol mandated follow-up were excluded from KCHF registry participants $(\mathrm{N}=4056)$. The participating physicians judged that it was difficult for the patients to visit 19 participating hospitals and undergo laboratory tests and echocardiography at $6 \pm 1$ months because of poor compliance with follow-up and HF management, cognitive dysfunction, frailty or functional disability or because they were discharged to and were mainly followed by primary-level hospitals and clinics, nursing care facilities and facilities offering long-term medical care or treatment unconnected with the participating hospitals or moved to a distant area, respectively. The mortality during 6 months after index hospitalisation $(1.8 \%)$ (figure 1 ) and in the ensuing 180 days after 6-month follow-up echocardiography $(4.6 \%-8.6 \%)$ (table 3) was much lower than the 180-day mortality of the ASCEND-HF (Acute Study of Clinical Effectiveness of Nesiritide in Decompensated Heart Failure) and trial-eligible GWTG-HF (Get With The GuidelinesHeart Failure) cohorts (18.6\% and 21.2\%). ${ }^{33}$ Indeed, the 180-day mortality of the 1516 patients ineligible for the protocol mandated follow-up (15.0\%) (data not shown) was comparable to that of the previous trials. In addition, many other patients were excluded at each stage (see figure 1). This study population only comprised 673 patients of the 4056 patients enrolled in the KCHF registry or of the 1246 patients scheduled for a 6-month echocardiography. This very significant selection of patients remains a major limitation to the present study. Further studies are needed to generalise our study results. Finally, there may be residual and unmeasured confounding factors related to outcomes, although we adjusted for 15 variables relevant to $\mathrm{HF}$ outcomes.

\section{CONCLUSION}

Patients with reduction in LAD during follow-up after ADHF hospitalisation had a lower risk for a composite of all-cause death or HF hospitalisation, suggesting that the 
change of LAD might be a simple and useful echocardiographic marker during follow-up.

\section{Author affiliations}

${ }^{1}$ Department of Cardiovascular Medicine, Kyoto University Graduate School of Medicine, Kyoto, Japan

${ }^{2}$ Department of Clinical Epidemiology, Hyogo College of Medicine, Nishinomiya, Japan

${ }^{3}$ Department of Cardiology, Mitsubishi Kyoto Hospital, Kyoto, Japan

${ }^{4}$ Cardiovascular Medicine, Shiga General Hospital, Moriyama, Japan

${ }^{5}$ Division of Cardiology, Tenri Hospital, Tenri, Japan

${ }^{6}$ Department of Cardiovascular Medicine, Kobe City Medical Center General

Hospital, Kobe, Japan

${ }^{7}$ Department of Cardiology, National Hospital Organization Kyoto Medical Center, Kyoto, Japan

${ }^{8}$ Department of Cardiology, Osaka Red Cross Hospital, Osaka, Japan

${ }^{9}$ Department of Cardiology, Kurashiki Central Hospital, Kurashiki, Japan

${ }^{10}$ Department of Cardiology, Kokura Memorial Hospital, Kokura, Japan

${ }^{11}$ Department of Cardiology, Japanese Red Cross Wakayama Medical Center,

Wakayama, Japan

${ }^{12}$ Department of Cardiology, Hyogo Prefectural Amagasaki General Medical Center, Amagasaki, Japan

${ }^{13}$ Department of Cardiovascular Medicine, Shinshu University Graduate School of Medicine, Nagano, Japan

Acknowledgements The authors thank the staff of the KCHF study, the other members of the participating centres.

Contributors Conceived the design: MS and TK. Collected data: HY, YI, YT, NO, YSe, EY, YYo, TK, YYa, MI, KN, YK, TM, MT, YF, KA, KKa, YSa and KKu. Performed statistical analysis: MS, TK, TM. Wrote the manuscript: MS and TK. Made critical revision: HY, YI, YT, NO, YSe, EY, YYo, TK, YYa, MI, KN, YK, TM, MT, YF, KA, KKa, YSa, KKu, TM and TK. Supervised: TM and TK.

Funding This work was supported by the Japan Agency for Medical Research and Development (18059186) (Drs T. Kato, Kuwahara and Ozasa).

Competing interests None declared.

Patient consent for publication Not required.

Ethics approval The study protocol was approved by the ethical committees at the Kyoto University Hospital (local identifier: E2311) and at each participating hospital.

Provenance and peer review Not commissioned; externally peer reviewed.

Data availability statement All data relevant to the study are included in the article or uploaded as supplementary information.

Supplemental material This content has been supplied by the author(s). It has not been vetted by BMJ Publishing Group Limited (BMJ) and may not have been peer-reviewed. Any opinions or recommendations discussed are solely those of the author(s) and are not endorsed by BMJ. BMJ disclaims all liability and responsibility arising from any reliance placed on the content. Where the content includes any translated material, BMJ does not warrant the accuracy and reliability of the translations (including but not limited to local regulations, clinical guidelines, terminology, drug names and drug dosages), and is not responsible for any error and/or omissions arising from translation and adaptation or otherwise.

Open access This is an open access article distributed in accordance with the Creative Commons Attribution Non Commercial (CC BY-NC 4.0) license, which permits others to distribute, remix, adapt, build upon this work noncommercially, and license their derivative works on different terms, provided the original work is properly cited, appropriate credit is given, any changes made indicated, and the use is non-commercial. See: http://creativecommons.org/ licenses/by-nc/4.0/.

\section{ORCID iDs}

Takao Kato http://orcid.org/0000-0001-8213-7999

Takeshi Morimoto http://orcid.org/0000-0002-6844-739X

Moritake Iguchi http://orcid.org/0000-0001-5029-1203

Kazuya Nagao http://orcid.org/0000-0002-5043-8778

Takeshi Kimura http://orcid.org/0000-0002-5665-4076
REFERENCES

1 Shimokawa $\mathrm{H}$, Miura M, Nochioka $\mathrm{K}$, et al. Heart failure as a general pandemic in Asia. Eur J Heart Fail 2015;17:884-92.

2 Ambrosy AP, Fonarow GC, Butler J, et al. The global health and economic burden of hospitalizations for heart failure: lessons learned from hospitalized heart failure registries. J Am Coll Cardiol 2014;63:1123-33.

3 Su K, Kato T, Toyofuku M, et al. Association of previous hospitalization for heart failure with increased mortality in patients hospitalized for acute decompensated heart failure. Circ Rep 2019;1:517-24.

4 Yaku H, Kato T, Morimoto T, et al. Risk factors and clinical outcomes of functional decline during hospitalisation in very old patients with acute decompensated heart failure: an observational study. BMJ Open 2020;10:e032674.

5 St John Sutton M, Linde C, Gold MR, et al. Left ventricular architecture, long-term reverse remodeling, and clinical outcome in mild heart failure with cardiac resynchronization: results from the REVERSE trial. JACC Heart Fail 2017;5:169-78.

6 Rossi A, Temporelli PL, Quintana M, et al. Independent relationship of left atrial size and mortality in patients with heart failure: an individual patient meta-analysis of longitudinal data (merge heart failure). Eur $J$ Heart Fail 2009;11:929-36.

7 Barasch E, Petillo F, Pollack S, et al. Clinical and echocardiographic correlates of mortality in medically treated patients with severe isolated aortic stenosis and normal left ventricular ejection fraction. Circ J 2014;78:232-9.

8 Spirito P, Autore C, Formisano F, et al. Risk of sudden death and outcome in patients with hypertrophic cardiomyopathy with benign presentation and without risk factors. Am J Cardiol 2014;113:1550-5.

9 Benjamin EJ, D'Agostino RB, Belanger AJ, et al. Left atrial size and the risk of stroke and death. The Framingham heart study. Circulation 1995;92:835-41.

10 Yamamoto E, Kato T, Ozasa N, et al. Kyoto congestive heart failure (KCHF) study: rationale and design. ESC Heart Fail 2017;4:216-23.

11 Yaku H, Ozasa N, Morimoto T, et al. Demographics, management, and in-hospital outcome of hospitalized acute heart failure syndrome patients in contemporary real clinical practice in Japan-observations from the prospective, multicenter Kyoto congestive heart failure (KCHF) registry. Circ J 2018;82:2811-9.

12 Yaku H, Kato T, Morimoto T, et al. Association of mineralocorticoid receptor antagonist use with all-cause mortality and hospital readmission in older adults with acute decompensated heart failure. JAMA Netw Open 2019;2:e195892.

13 Kitai T, Miyakoshi C, Morimoto T, et al. Mode of death among Japanese adults with heart failure with preserved, Midrange, and reduced ejection fraction. JAMA Netw Open 2020;3:e204296.

14 Kato T, Yaku H, Morimoto T, et al. Association with controlling nutritional status (CONUT) score and in-hospital mortality and infection in acute heart failure. Sci Rep 2020;10:3320.

15 Yamamoto E, Kato T, Yaku H, et al. Sex differences in patients with acute decompensated heart failure in Japan: observation from the KCHF registry. ESC Heart Fail 2020;7:2485-2493.

16 Dandel $\mathrm{M}$, Weng $\mathrm{Y}$, Siniawski $\mathrm{H}$, et al. Heart failure reversal by ventricular unloading in patients with chronic cardiomyopathy: criteria for weaning from ventricular assist devices. Eur Heart $J$ 2011;32:1148-60.

17 Nagueh SF, Smiseth OA, Appleton CP, et al. Recommendations for the evaluation of left ventricular diastolic function by echocardiography: an update from the american society of echocardiography and the European association of cardiovascular imaging. J Am Soc Echocardiogr 2016;29:277-314.

18 Rusinaru D, Tribouilloy C, Grigioni F, et al. Left atrial size is a potent predictor of mortality in mitral regurgitation due to flail leaflets: results from a large international multicenter study. Circ Cardiovasc Imaging 2011;4:473-81.

19 Beinart R, Boyko V, Schwammenthal E, et al. Long-Term prognostic significance of left atrial volume in acute myocardial infarction. $J$ Am Coll Cardiol 2004;44:327-34.

20 Vaziri SM, Larson MG, Benjamin EJ, et al. Echocardiographic predictors of nonrheumatic atrial fibrillation. The Framingham heart study. Circulation 1994;89:724-30.

21 Meris A, Amigoni $\mathrm{M}, \mathrm{Uno} \mathrm{H}$, et al. Left atrial remodelling in patients with myocardial infarction complicated by heart failure, left ventricular dysfunction, or both: the VALIANT echo study. Eur Heart J 2009;30:56-65

22 Lang RM, Badano LP, Mor-Avi V, et al. Recommendations for cardiac chamber quantification by echocardiography in adults: an update from the American Society of echocardiography and the European association of cardiovascular imaging. Eur Heart $J$ Cardiovasc Imaging 2015;16:233-71. 
23 Lang RM, Badano LP, Mor-Avi V, et al. Recommendations for cardiac chamber quantification by echocardiography in adults: an update from the American Society of echocardiography and the European association of cardiovascular imaging. $J$ of the Echocardiogr 2015;28:1-39.

24 Hoit BD. Left atrial size and function: role in prognosis. J Am Coll Cardiol 2014;63:493-505.

25 Oliver W, Matthews G, Ayers CR. Factors associated with left atrial remodeling in the general population. Circulation 2017;10.

26 Appleton CP, Galloway JM, Gonzalez MS, et al. Estimation of left ventricular filling pressures using two-dimensional and Doppler echocardiography in adult patients with cardiac disease. Additional value of analyzing left atrial size, left atrial ejection fraction and the difference in duration of pulmonary venous and mitral flow velocity at atrial contraction. J Am Coll Cardiol 1993;22:1972-82.

27 Yeboah J, Bluemke DA, Hundley WG, et al. Left ventricular dilation and incident congestive heart failure in asymptomatic adults without cardiovascular disease: multi-ethnic study of atherosclerosis (MESA) J Card Fail 2014;20:905-11.

28 Froehlich L, Meyre P, Aeschbacher S, et al. Left atrial dimension and cardiovascular outcomes in patients with and without atrial fibrillation: a systematic review and meta-analysis. Heart 2019;105:1884-91.

29 Curtis JP, Sokol SI, Wang Y, et al. The association of left ventricular ejection fraction, mortality, and cause of death in stable outpatients with heart failure. J Am Coll Cardiol 2003;42:736-42.

30 Collier P, Watson CJ, Waterhouse DF, et al. Progression of left atria volume index in a population at risk for heart failure: a substudy of the STOP-HF (ST Vincent's screening to prevent heart failure) trial. Eur J Heart Fail 2012;14:957-64.

31 Cameli M, Sciaccaluga C, Loiacono F, et al. The analysis of left atrial function predicts the severity of functional impairment in chronic heart failure: the flash multicenter study. Int $J$ Cardiol 2019;286:87-91.

32 Cho G-Y, Marwick TH, Kim H-S, et al. Global 2-dimensional strain as a new prognosticator in patients with heart failure. J Am Coll Cardiol 2009;54:618-24.

33 Greene SJ, DeVore AD, Sheng S, et al. Representativeness of a heart failure trial by race and sex: results from ASCEND-HF and GWTG-HF. JACC Heart Fail 2019;7:980-92. 